\title{
Genome-wide expression profiling of the response to short-term exposure to fluconazole in Cryptococcus neoformans serotype A
}

\author{
Ada Rita Florio ${ }^{1}$, Selene Ferrari ${ }^{2}$, Elena De Carolis ${ }^{1}$, Riccardo Torelli ${ }^{1}$, Giovanni Fadda ${ }^{1}$, Maurizio Sanguinetti ${ }^{*}$,
} Dominique Sanglard ${ }^{2}$ and Brunella Posteraro ${ }^{1}$

\begin{abstract}
Background: Fluconazole ( $F L C)$, a triazole antifungal drug, is widely used for the maintenance therapy of cryptococcal meningoencephalitis, the most common opportunistic infection in AIDS patients. In this study, we examined changes in the gene expression profile of the C. neoformans reference strain H99 (serotype A) following FLC treatment in order to investigate the adaptive cellular responses to drug stress.

Results: Simultaneous analysis of over 6823 transcripts revealed that 476 genes were responsive to FLC. As expected up-regulation of genes involved in ergosterol biosynthesis was observed, including the azole target gene ERG11 and ERG13, ERG1, ERG7, ERG25, ERG2, ERG3 and ERG5. In addition, SRE1 which is a gene encoding a wellknown regulator of sterol homeostasis in C. neoformans was up-regulated. Several other genes such as those involved in a variety of important cellular processes (i.e. lipid and fatty acid metabolism, cell wall maintenance, stress and virulence) were found to be up-regulated in response to FLC treatment. Conversely, expression of AFR1, the major transporter of azoles in C. neoformans, was not regulated by FLC.

Conclusions: Short-term exposure of C. neoformans to FLC resulted in a complex altered gene expression profile. Some of the observed changes could represent specific adaptive responses to the antifungal agent in this pathogenic yeast.
\end{abstract}

\section{Background}

Cryptococcus neoformans is a basidiomycetous fungal pathogen that causes meningoencephalitis in predominantly immunocompromised hosts [1,2], that is the most devastating manifestation of cryptococcal disease and is fatal unless treated [3]. Cryptococcosis appears to be a significant opportunistic infection in solid-organ transplant recipients, with a prevalence rate ranging from $0.26 \%$ to $5 \%$ and overall mortality of $42 \%$ [4]. Notably, cryptococcal meningitis was reported to occur in $46 \%$ of patients from an Indian HIV-positive cohort [5]. Although the introduction of highly active antiretroviral therapy has led to a decrease in the number of cryptococcal infections in AIDS patients in most developed countries, this is not the case in developing countries where the incidence of HIV/AIDS

\footnotetext{
* Correspondence: msanguinetti@rm.unicatt.it

${ }^{1}$ Istituto di Microbiologia, Università Cattolica del Sacro Cuore, Largo F. Vito 1, 00168 Rome, Italy

Full list of author information is available at the end of the article
}

and cryptococcal meningitis continue to rise [6]. As fluconazole (FLC) became increasingly used due to the need for life-long maintenance therapy in HIV/AIDS patients, FLC resistance was hence detected at relatively high frequency in C. neoformans clinical isolates from India, Africa and Cambodia [7-9].

Increased FLC resistance in vitro was shown to be predictive of treatment failures and infection relapses [10]. Recently, the mechanism underlying the heteroresistance to FLC was elucidated [11], that is an adaptive mode of azole resistance previously associated with FLC therapy failure cases [12]. This mechanism is based on duplications of multiple chromosomes in response to drug pressure [13]. Interestingly, Sionov et al. [13] observed that the number of disomic chromosomes positively correlated with the duration of exposure to FLC, whereas the duplication of chromosome 1 was closely associated with two genes, ERG11, the target of FLC [14], and AFR1, the major transporter of azoles in C. neoformans $[11,15]$. Such genomic plasticity
C Biomed Central

() 2011 Florio et al; licensee BioMed Central Ltd. This is an Open Access article distributed under the terms of the Creative Commons Attribution License (http://creativecommons.org/licenses/by/2.0), which permits unrestricted use, distribution, and reproduction in any medium, provided the original work is properly cited. 
enables cells to cope with drug stress and was observed in C. neoformans strains of both serotypes, A (C. neoformans var. grubii) and D (C. neoformans var. neoformans) [13].

The recent sequencing of the $C$. neoformans genome [16] has stimulated the development of C. neoformansspecific microarrays that made possible to address hypotheses about global responses to overcome stresses during growth in the human host $[17,18]$. Regardless of the source (i.e. host-derived or antifungal drugs), toxic compounds exert constant selective pressure on the fungus that responds by developing mechanisms necessary for survival [19].

With the aim to identify genes required for adaptive growth in the presence of sub-inhibitory concentrations of FLC, we investigated here the transient response of C. neoformans to FLC by analyzing differences in gene expression prior and after FLC exposure of strain H99, a reference strain of serotype A. Thus, genome-wide transcriptional profiling of over 6823 C. neoformans genes identified 476 genes with significant expression changes. Apart from genes involved in ergosterol biosynthesis (e.g. ERG11), genes involved in other important cellular functions, such as those encoding the sterol homeostasis regulator Sre1 [20] or phospholipase B1 (Plb1) [21], were shown to be induced by FLC treatment. In addition, AFR 1 was not found FLC-responsive, suggesting indirectly that this gene is responsible for long-term FLC adaptation in C. neoformans.

\section{Methods}

\section{Strain, growth conditions and RNA isolation}

C. neoformans var. grubii serotype A strain (H99) was obtained from David S. Perlin [22], kept as 20\% glycerol stock at $-80^{\circ} \mathrm{C}$ and sub-cultured, as required, on YEPD (1\% yeast extract, $2 \%$ peptone, $2 \%$ glucose) agar plates at $30^{\circ} \mathrm{C}$. For RNA isolation independent overnight cultures were diluted 1:100 in liquid YEPD and grown at $30^{\circ} \mathrm{C}$ or $37^{\circ} \mathrm{C}$ with agitation for $3 \mathrm{~h}$ to reach a density of $3 \times 10^{7} \mathrm{CFU} / \mathrm{ml}$. At this point cultures were equally divided into two aliquots to which either FLC at a concentration of $10 \mathrm{mg} / \mathrm{l}$ or distilled water was added, followed by incubation at $30^{\circ} \mathrm{C}$ or $37^{\circ} \mathrm{C}$ for $90 \mathrm{~min}$. After this treatment, cultures were centrifuged at $4^{\circ} \mathrm{C}$ and $5500 \times g$ and total RNA was extracted as previously described [23].

\section{Microarray design and preparation}

C. neoformans H99 microarrays were designed following the Agilent Array Design guidelines (Earray platform) by first creating two separate sets of 60-base nucleotide probes for each of 6967 open reading frame (ORF) sequences as downloaded from the Broad Institute website http://www.broadinstitute.org/annotation/genome/ cryptococcusneoformans/MultiHome.html. The probe selection was performed using the GE Probe Design
Tool; probes were filtered following their base composition and distribution, cross-hybridization potential, and melting temperature, to yield final duplicate probes representing 6823 ORFs to cover $97.9 \%$ of the whole C. neoformans $\mathrm{H} 99$ genome. C. neoformans custom arrays were manufactured in the $8 \times 15 \mathrm{k}$ format by Agilent Technologies (Santa Clara, CA, USA). For quality control and normalization purposes, 157 probes were selected randomly and spotted 10 times throughout each array. Standard controls (Agilent Technologies) were also included.

\section{cRNA synthesis, labeling and hybridization}

RNA sample preparation was performed on three biological triplicates of $\mathrm{H} 99$ cells grown at $30^{\circ} \mathrm{C}$, as described above. Prior to the labeling/amplification step, purity and integrity of the RNA samples were determined using Agilent RNA 6000 Nano LabChip kit on the Agilent 2100 bioanalyzer (Agilent Technologies). Agilent's One-Color Quick Amp Labeling kit (Agilent Technologies) was used to generate fluorescently labeled cRNA probes according to the manufacturer's instructions. The method uses T7 RNA polymerase, which simultaneously amplifies target material and incorporates cyanine 3-labeled CTP. The labeled cRNAs were purified with the RNeasy Mini kit (Qiagen, Hilden, Germany) and quantified using NanoDrop ND-1000 UV-VIS spectrophotometer. Aliquots (600 ng) of Cy3-labeled cRNAs were fragmented and hybridized for $17 \mathrm{~h}$ at $65^{\circ} \mathrm{C}$ to each array using the Gene Expression Hybridization kit (Agilent Technologies) and according to the manufacturer's instructions.

\section{Microarray imaging and data analysis}

Slides were washed and processed according to the Agilent 60-mer Oligo Microarray Processing protocol and scanned on a Agilent microarray scanner G2565BA (Agilent Technologies). Data were extracted from the images with Feature Extraction (FE) software (Agilent Technologies). FE software flags outlier features, and detects and removes spatial gradients and local backgrounds. Data were normalized using a combined rank consistency filtering with LOWESS intensity normalization. The gene expression values obtained from FE software were imported into GeneSpring 10.0.2 software (Agilent Technologies) for pre-processing and data analysis. For interarray comparisons, a linear scaling of the data was performed using the 75th percentile signal value of all of non-control probes on the microarray to normalize onecolour signal values. Probe sets with a signal intensity value below the 20th percentile were considered as absent and discarded from subsequent analysis. The expression of each gene was normalized by its median expression across all samples. Genes were included in the final data set if their expression changed by at least 
twofold between strain H99 FLC-exposed or -not exposed (control sample) in at least two independent experiments, together with a $P$-value cut-off of $<0.05$ (by one-way analysis of variance [ANOVA] corrected). Genes listed in Table 1 were categorized by reported or putative functions by the BROAD Institute database with NCBI blastP http://www.ncbi.nlm.nih.gov/BLAST/ editing, and also by the Uniprot http://www.uniprot.org/ and Saccharomyces genome http://www.yeastgenome.org/cgibin/blast-sgd.pl databases. As indicated in Table 1, each $S$. cerevisiae gene name was assigned by blastP search with the $C$. neoformans $\mathrm{H} 99$ gene sequence ( $e$-value cutoff: $\mathrm{e}^{-6}$ ) according to Kim et al. [24]. Gene Ontology (GO) term analysis was carried to help categorize a list of genes into functional groups. The whole microarray data have been deposited in National Center for Biotechnology Information's Gene Expression Omnibus [25] and are accessible through GEO Series accession number GSE24927.

\section{Quantitative RT-PCR (qRT-PCR) validation of gene expression}

Expression of selected differentially regulated genes as identified by the microarray analysis was quantitatively assessed with qRT-PCR in an i-Cycler iQ system (BioRad Laboratories, Hercules, CA, USA). All primers and probes (see Additional file 1) were designed with Beacon Designer 2 (version 2.06) software (Premier Biosoft International, Palo Alto, CA, USA) and synthesized by MWG Biotech (Florence, Italy). qRT-PCRs were carried out as previously described [23]. The annealing temperature used for all primers was $65^{\circ} \mathrm{C}$. Each reaction was run in triplicate on three separate occasions. For relative quantification of target gene expression, $A C T 1$ was used as a normalizer gene [23]. Changes ( $n$-fold) in gene expression relative to that of the control were determined from mean ACT1-normalized expression levels.

\section{Oxidative stress and cell wall inhibitor assays}

Susceptibilities to hydrogen peroxide $\left(\mathrm{H}_{2} \mathrm{O}_{2}\right)$ and cell wall inhibitors were measured with exponentially growing cells in liquid YEPD at $30^{\circ} \mathrm{C}$ or $37^{\circ} \mathrm{C}$ pre-treated or not with FLC $(10 \mathrm{mg} / \mathrm{l})$ for $90 \mathrm{~min}$ as described elsewhere with modifications [26,27]. The cells were next washed with sterile PBS and diluted to an $\mathrm{OD}_{650}$ of 1.0 in PBS. For the oxidative stress assays, aliquots of the cell suspensions were transferred to Eppendorf tubes where $\mathrm{H}_{2} \mathrm{O}_{2}$ (Sigma, Milan, Italy) was added to $20 \mathrm{mM}$ and incubated at $30^{\circ} \mathrm{C}$ or $37^{\circ} \mathrm{C}$ for $2 \mathrm{~h}$. Viability was determined after appropriate dilution of the samples with PBS by plating $100 \mu \mathrm{l}$ in triplicate on solid YEPD. The CFU were counted after incubation for $72 \mathrm{~h}$ at $30^{\circ}$ $\mathrm{C}$ or $37^{\circ} \mathrm{C}$. For the cell wall inhibitor assays, dilutions of the cell suspensions were made in PBS and $5 \mu \mathrm{l}$ of these were grown on YEPD plates containing 0.5\% Congo red (Sigma, C-6767), 0.5, 1.0 and $1.5 \mathrm{mg} \mathrm{ml}^{-1}$ calcofluor white (Sigma, F-3543), 0.01\%, 0.03\% and 0.06\% SDS (Sigma) and 0.2, 0.5 and $1.0 \mathrm{mg} \mathrm{ml}^{-1}$ caffeine (Sigma, C0750). Plates were incubated for $48 \mathrm{~h}$ at $30^{\circ} \mathrm{C}$ or $37^{\circ} \mathrm{C}$ and photographed.

\section{Results and Discussion}

Experimental design and global gene expression results The transcript profiles of C. neoformans H99 cells exposed to $10 \mathrm{mg} / \mathrm{l}$ of FLC $(1 / 2 \times \mathrm{MIC})$ for one doubling time $(90 \mathrm{~min})$ at $30^{\circ} \mathrm{C}$ were compared with profiles of untreated cells. A total of 476 genes were found responsive to FLC treatment under the test conditions, consisting of a single concentration and a single time point as described elsewhere [28-30]. The threshold value used in the present analysis was at least a twofold difference of gene expression between the experimental conditions, which is a value generally accepted in fungal genome-wide expression profiling [31]. Given that approximately $95 \%$ of the genes $(6434 / 6823)$ spotted on the microarrays gave validated data, the above mentioned number indicate that $7.4 \%$ of the total number of genes in the C. neoformans $\mathrm{H} 99$ genome exhibited transcriptional changes, with 231 genes being upregulated and 245 downregulated upon FLC treatment.

In order to verify the changes in gene expression identified by our microarray analysis, we randomly selected 10 target genes (CNAG_00747, CNAG_01858, CNAG_02048, CNAG_02226, CNAG_03007, CNAG_03204, CNAG_ 04632, CNAG_03433,CNAG_05264,CNAG_05602) including those regulated and not regulated by FLC for validation of microarray data. A strong correlation $(\mathrm{r}=$ 0.94) was found between relative expression levels obtained by microarray or qRT-PCR analysis (Figure 1). In addition, qRT-PCR experiments performed with RNA extracted from $\mathrm{H} 99$ cells $\mathrm{FLC}$-treated at $37^{\circ} \mathrm{C}$ demonstrated that expression of the target genes also including AFR 1 was comparable to that obtained when $\mathrm{H} 99$ cells were pre-treated with FLC at $30^{\circ} \mathrm{C}$ (Figure 2).

The genes listed in Table 1 were categorized in 10 main groups by functional profiles as described in Methods. The category with the largest number of genes was "transport" with 31 genes, followed by categories that include genes $(n=18)$ involved in carbohydrate metabolism or protein processes (i.e. biosynthesis, modification, transport and degradation). While up- or down-regulated genes were distributed homogenously within almost all the function groups, some categories included more up-regulated genes (ergosterol biosynthesis) or down-regulated genes (TCA cycle). As it will be discussed below, the finding of a large number of genes differentially regulated adds support to the concept that azole activity is beyond the inhibition of the lanosterol demethylase target encoded by ERG11 
Table 1 Changes in the gene expression of C. neoformans $\mathrm{H} 99$ cells exposed to FLC

\begin{tabular}{|c|c|c|c|c|}
\hline $\begin{array}{c}\text { BROAD ID } \\
\text { (CNAG_*****) }\end{array}$ & $\begin{array}{l}\text { C. } n \text {. gene } \\
\text { name }\end{array}$ & $\begin{array}{l}\text { S. c. gene } \\
\text { name }\end{array}$ & Description & $\begin{array}{c}\text { Fold } \\
\text { change }\end{array}$ \\
\hline \multicolumn{5}{|c|}{ Ergosterol biosynthesis } \\
\hline 04804 & SRE1 & & Sterol regulatory element-binding protein 1 & +4.04 \\
\hline 01737 & & ERG25 & C-4 methyl sterol oxidase & +3.95 \\
\hline 00854 & & $E R G 2$ & C-8 sterol isomerase & +3.47 \\
\hline 02896 & & ERG13 & Hydroxymethylglutaryl-CoA synthase & +3.03 \\
\hline 06644 & & ERG5 & C-22 sterol desaturase & +2.50 \\
\hline 00040 & $E R G 11$ & ERG11 & Lanosterol 14 alpha-demethylase & +2.47 \\
\hline 06829 & & ERG1 & Squalene monooxygenase & +2.37 \\
\hline 00519 & & ERG3 & C-5 sterol desaturase & +2.21 \\
\hline 01129 & & ERG7 & Lanosterol synthase & +2.09 \\
\hline \multicolumn{5}{|l|}{ Transport } \\
\hline 04632 & & FUR4 & Uracil permease & +5.87 \\
\hline 07448 & & DUR3 & Urea transporter & +4.78 \\
\hline 04758 & & MEP2/AMP2 & Ammonium transporter & +3.78 \\
\hline 06652 & & DAL5 & Allantoate permease & +2.83 \\
\hline 01742 & & AQY1 & Water channel & +2.73 \\
\hline 07902 & & CAN1 & Amino acid transporter & +2.52 \\
\hline 01960 & & YMR279C & Efflux protein EncT & +2.47 \\
\hline 06338 & & PDR15 & ABC transporter PMR5 & +2.37 \\
\hline 04898 & & ATR1 & MFS transporter & +2.37 \\
\hline 00284 & & YOR378W & Efflux protein EncT & +2.36 \\
\hline 00097 & & ITR1 & ITR1 & +2.26 \\
\hline 00895 & & ZRT1 & Low-affinity zinc ion transporter & +2.20 \\
\hline 04210 & & $\mathrm{MPH} 2$ & Sugar transporter & +2.15 \\
\hline 04617 & & OPT2 & Small oligopeptide transporter & +2.11 \\
\hline 05592 & & PMR1 & Calcium-transporting ATPase & +2.06 \\
\hline 01059 & & YBR241C & Vacuolar membrane protein & +2.02 \\
\hline 00904 & & $A Z R 1$ & Aflatoxin efflux pump AFLT & -2.10 \\
\hline 01769 & & AGC1 & Mitochondrial inner membrane protein & -2.16 \\
\hline 04142 & & FEN2 & Tartrate transporter & -2.17 \\
\hline 04567 & & TPO2 & Drug transporter & -2.22 \\
\hline 05387 & & HXT5 & Galactose transporter & -2.28 \\
\hline 02355 & & YEA4 & UDP-N-acetylglucosamine transporter & -2.30 \\
\hline 05994 & & FLR1 & Multidrug transporter & -2.35 \\
\hline 02733 & & STL1 & Hexose transport-related protein & -2.46 \\
\hline 03794 & & YBR287W & Endoplasmic reticulum protein & -2.58 \\
\hline 00815 & & SIT1 & Siderochrome-iron (Ferrioxamine) uptake transporter & -2.92 \\
\hline 01354 & & TNA1 & Transporter & -3.39 \\
\hline 02104 & SFH5 & SFH5 & Phosphatidylinositol transfer protein SFH5 & -4.54 \\
\hline 07695 & & UGA4 & Gamma-aminobutyric acid transporter & -5.16 \\
\hline 00749 & & YIL166C & Transporter & -5.65 \\
\hline 02083 & & ARN2 & Siderochrome-iron transporter & -9.48 \\
\hline \multicolumn{5}{|l|}{ Cell wall maintenance } \\
\hline 02217 & & CHS7 & Chitin synthase 7 & +3.62 \\
\hline 06336 & & $B G L 2$ & Glucan 1,3 beta-glucosidase protein & +2.61 \\
\hline 03326 & & CHS2 & Chitin synthase 2 , CHS2 & +2.20 \\
\hline 01239 & CDA3 & CDA2 & Chitin deacetylase & -4.35 \\
\hline
\end{tabular}


Table 1 Changes in the gene expression of C. neoformans H99 cells exposed to FLC (Continued)

$\begin{array}{ccc}03644 & \text { CAS3 } & \\ 01489 & \text { CAS9 } & \text { YJL218W } \\ & & \\ \text { The fatty acid metabolism } & & \text { PLB1 } \\ 06085 & \text { PLB1 } & \\ 06623 & \text { MIOX } & \text { ECM38 } \\ 03128 & & \text { PCT1 } \\ 00424 & & \text { CAT2 } \\ 05042 & & \text { FOX2 } \\ 02000 & & \text { PSD2 } \\ 00834 & & \\ 02968 & \text { PLC2 } & \end{array}$

CAS3p
Putative O-acetyl transferase

Lipid and fatty acid metabolism

$\begin{array}{cc}\text { Phospholipase B } & +2.18 \\ \text { Myo-inositol oxygenase } & +2.12 \\ \text { Lincomycin-condensing protein ImbA } & -2.01 \\ \text { Choline-phosphate cytidylyltransferase } & -2.02 \\ \text { Carnitine acetyltransferase } & -2.10 \\ \text { Short-chain dehydrogenase } & -2.95 \\ \text { Phosphatidylserine decarboxylase } & -3.10 \\ \text { Phospholipase C-2 } & -4.11\end{array}$

Cell stress

$\begin{array}{lcc}03400 & & \text { GRE2 } \\ 05256 & \text { CTA1 } \\ 02440 & & \text { HSC82 } \\ 01750 & \text { SSA1 } \\ 06917 & \text { PRX1 } \\ 03185 & \text { TSA3 } & \text { LOT6 } \\ 04622 & & \text { SNG1 } \\ 00575 & & \text { CTT1 } \\ 01464 & & \text { YHB1 }\end{array}$

$\begin{array}{cc}\text { Oxidoreductase } & +3.54 \\ \text { Catalase 2 } & +2.81 \\ \text { Cation-transporting ATPase } & +2.54 \\ \text { Heat shock protein } 70 & +2.48 \\ \text { Thiol-specific antioxidant protein 3 } & +2.09 \\ \text { Low temperature-responsive protein } & +2.05 \\ \text { Response to drug-related protein } & -2.17 \\ \text { Catalase } & -2.21 \\ \text { Flavo-haemoglobin } & -2.32\end{array}$

Amino acid metabolism

$\begin{array}{lc}02284 & \text { PDA1 } \\ 04862 & \text { GLT1 } \\ 04017 & \text { MXR2 } \\ 01231 & \text { CAR1 } \\ 03828 & \text { ARO8 } \\ 06540 & \text { ILV3 } \\ 00247 & \text { LYS9 } \\ 02270 & \text { MET2 } \\ 01076 & \text { UGA1 } \\ 00237 & \text { LEU1 } \\ 01264 & \text { LYS12 } \\ 00879 & \text { GDH2 } \\ 04467 & \text { UGA2 } \\ 02851 & \text { GLY1 } \\ 02049 & \text { PUT1 } \\ 05602 & \text { PUT2 }\end{array}$

$\begin{array}{cc}\text { Branched-chain alpha-keto acid dehydrogenase E1-alpha subunit } & +2.42 \\ \text { Glutamate synthase (NADH) } & +2.39 \\ \text { Protein-methionine-R-oxide reductase } & +2.32 \\ \text { Arginase } & +2.27 \\ \text { Aromatic amino acid aminotransferase I } & +2.26 \\ \text { Dihydroxy-acid dehydratase } & +2.18 \\ \text { Saccharopine dehydrogenase (NADP+, L-glutamate-forming) } & +2.02 \\ \text { Homoserine O-acetyltransferase } & -2.11 \\ \text { 4-aminobutyrate transaminase } & -2.18 \\ \text { 3-isopropylmalate dehydratase } & -2.27 \\ \text { Isocitrate dehydrogenase } & -2.31 \\ \text { Glutamate dehydrogenase } & -2.33 \\ \text { Threonine aldolase } & -2.83 \\ \text { Proline dehydrogenase } & -3.04 \\ \text { 1-pyrroline-5-carboxylate dehydrogenase } & -5.74 \\ \end{array}$

Carbohydrate metabolism

$\begin{array}{lc}06374 & \text { MAE1 } \\ 02225 & \text { CELC } \\ 02552 & \text { TKL1 } \\ 04025 & \text { TAL1 } \\ 00696 & \text { AMS1 } \\ 05913 & \text { MAL12 } \\ 05113 & \text { ALD4 } \\ 05264 & \text { YJL216C } \\ 03946 & \text { GAL1 }\end{array}$

$\begin{array}{cc}\text { Malic enzyme } & +6.04 \\ \text { Cellulase } & +3.99 \\ \text { Transketolase } & +3.28 \\ \text { Transaldolase } & +3.00 \\ \text { Alpha-mannosidase } & +2.52 \\ \text { Alpha-glucosidase } & +2.34 \\ \text { Aldehyde dehydrogenase (ALDDH) } & +2.11 \\ \text { Alpha-amylase AmyA } & +2.08 \\ \text { Galactokinase } & -2.16\end{array}$


Table 1 Changes in the gene expression of C. neoformans $\mathrm{H} 99$ cells exposed to FLC (Continued)

\begin{tabular}{|c|c|c|}
\hline 07752 & GLF & \\
\hline 04659 & & PDC1 \\
\hline 06924 & & SUC2 \\
\hline 00269 & & SOR1 \\
\hline 00393 & GLC3 & GLC3 \\
\hline 07745 & MPD1 & $\mathrm{ADH} 3$ \\
\hline 04217 & & PCK1 \\
\hline 04621 & & GSY1 \\
\hline 04523 & & $\mathrm{TDH} 3$ \\
\hline \multicolumn{3}{|c|}{ Protein biosynthesis, modification, transport, and degradation } \\
\hline 02389 & & YPK1 \\
\hline 02531 & & FUS3 \\
\hline 03176 & & $E R O 1$ \\
\hline 05932 & CPR6 & CPR6 \\
\hline 01861 & & NAS6 \\
\hline 04635 & & PEP4 \\
\hline 06872 & & YKL215C \\
\hline 05005 & ATG1 & ATG1 \\
\hline 00919 & & KEX1 \\
\hline 04625 & & PRB1 \\
\hline 00130 & & $R C K 2$ \\
\hline 04108 & & PKP1 \\
\hline 02327 & & YFR006W \\
\hline 02418 & & DED81 \\
\hline 03563 & & DPS1 \\
\hline 04275 & & OMA1 \\
\hline 02006 & & NTA1 \\
\hline 03949 & & $\mathrm{PHO} 13$ \\
\hline
\end{tabular}

$\begin{array}{cc}\text { UDP-galactopyranose mutase } & -2.23 \\ \text { Pyruvate decarboxylase } & -2.33 \\ \text { Beta-fructofuranosidase } & -2.57 \\ \text { Sorbitol dehydrogenase } & -2.62 \\ \text { 1,4-alpha-glucan-branching enzyme } & -2.93 \\ \text { Mannitol-1-phosphate dehydrogenase } & -3.54 \\ \text { Phosphoenolpyruvate carboxykinase } & -8.67 \\ \text { Glycogen (Starch) synthase } & -11.00 \\ \text { Glyceraldehyde-3-phosphate dehydrogenase } & -11.45\end{array}$

TCA cycle

$03596 \quad$ KGD2

$03920 \quad$ IDPI

$03674 \quad K G D 1$

$00747 \quad$ LSC2

$07363 \quad$ IDH2

01137 ACO1

$07851 \quad$ IDH1

$\begin{array}{cc}\text { AGC-group protein kinase } & +3.04 \\ \text { Mitogen-activated protein kinase CPK1 } & +2.91 \\ \text { Endoplasmic oxidoreductin 1 } & +2.36 \\ \text { Peptidyl-prolyl cis-trans isomerase D } & +2.35 \\ \text { Proteolysis and peptidolysis-related protein } & +2.35 \\ \text { Endopeptidase } & +2.31 \\ \text { 5-oxoprolinase } & +2.27 \\ \text { Serine/threonine-protein kinase ATG1 } & +2.20 \\ \text { Carboxypeptidase D } & +2.13 \\ \text { Serine-type endopeptidase } & -2.01 \\ \text { Serine/threonine-protein kinase } & -2.12 \\ \text { Kinase } & -2.17 \\ \text { Prolidase } & -2.28 \\ \text { Asparagine-tRNA ligase } & -2.40 \\ \text { Aspartate-tRNA ligase } & -2.50 \\ \text { Metalloendopeptidase } & -2.50 \\ \text { 4-nitrophenylphosphatase } & -2.75 \\ \text { Protein N-terminal asparagine amidohydrolase } & -3.32\end{array}$

$\begin{array}{cc}\text { 2-oxoglutarate metabolism-related protein } & -2.02 \\ \text { Isocitrate dehydrogenase (NADP+) } & -2.06 \\ \text { Oxoglutarate dehydrogenase (Succinyl-transferring) } & -2.52 \\ \text { Succinate-CoA ligase (ADP-forming) } & -2.70 \\ \text { Isocitrate dehydrogenase } & -2.80 \\ \text { Aconitase } & -2.99 \\ \text { Isocitrate dehydrogenase (NAD+), putative } & -3.80\end{array}$

Glycerol metabolism

$\begin{array}{ll}06132 & \text { RHR2 } \\ 02815 & \text { GUT2 }\end{array}$

Glycerol-1-phosphatase $\quad+2.31$

Glycerol-3-phosphate dehydrogenase $\quad-2.00$

Nucleotide metabolism

$\begin{array}{ll}05545 & \text { HNT2 } \\ 03078 & \text { NPP1 } \\ & \\ 06489 & \text { ADO1 } \\ 00613 & \text { FCY1 }\end{array}$

Nucleoside-triphosphatase $\quad+2.25$

Type I phosphodiesterase/nucleotide pyrophosphatase family $\quad+2.08$ protein

Adenosine kinase

$-2.08$

ADO 1
FCY1

Cytosine deaminase

$-2.69$

Thiamin metabolism 
Table 1 Changes in the gene expression of C. neoformans $\mathrm{H} 99$ cells exposed to FLC (Continued)

Alcohol metabolism

$\begin{array}{lll}05258 & \text { SMG1 } & \\ 05024 & & \text { SPS19 } \\ 06168 & \text { GNO1 } & \text { SFA1 }\end{array}$

Glucose-methanol-choline (GMC) oxidoreductase

06168

SFA1

GSNO reductase

Carbon utilization

05144

CAN2

NCE103

Carbonic anhydrase 2

Cell cycle control

$\begin{array}{ll}03385 & \text { PCL1 } \\ 02604 & \text { HOP1 } \\ 00995 & \text { MSC1 }\end{array}$

G1/s-specific cyclin pcl1 (Cyclin hcs26)

$+2.37$

Putative uncharacterized protein

$+2.19$

Meiotic recombination-related protein

$-3.63$

Chromatin and chromosome structures

$$
02115
$$

Transcription

$\begin{array}{lc}01841 & \text { GLN3 } \\ 02990 & \text { YORO52C } \\ 04594 & \text { UGA3 } \\ 05290 & \text { SPT3 } \\ 06495 & \text { RNH70 } \\ 05333 & \text { PUT3 } \\ 02338 & \text { GIS2 } \\ 05479 & \text { ASG1 }\end{array}$

Predicted protein

$+5.72$

Nucleus protein

$+2.16$

PRO1 protein

$-2.01$

Transcription cofactor

$-2.01$

Ribonuclease $\mathrm{H}$

$-2.06$

Putative uncharacterized protein

$-2.14$

DNA-binding protein hexbp

$-2.47$

Putative uncharacterized protein

$-3.57$

Signal transduction

$\begin{array}{lll}03316 & & \text { RDI1 } \\ 00363 & \text { HHK5 } & \text { SLN1 } \\ 01262 & \text { GPB1 } & \text { STE4 }\end{array}$

Rho GDP-dissociation inhibitor 1

CnHHK5 protein

$-2.44$

G-protein beta subunit GPB1

$-2.55$

Oxidoreduction

04652

YLR460C

06035

ADH1

00605

ZTA1

00038

SOR2

01954

YPR127W

02958

FET5

02935

YMR226C

XYL2

FRE7

MET10

YELO4TC

FRE2

AlF1

$+2.63$

Enoyl reductase

$+2.41$

Alcohol dehydrogenase

$+2.20$

Cytoplasm protein

$+2.13$

Alcohol dehydrogenase

$+2.09$

Aldo/keto reductase

$+2.06$

Ferroxidase

$-2.01$

Zinc-binding dehydrogenase

$-2.28$

Ferric-chelate reductase

$-2.49$

Sulfite reductase (NADPH)

$-2.55$

03168

Fumarate reductase (NADH)

$-2.58$

Metalloreductase

$-2.85$

03874

Oxidoreductase

Other 
Table 1 Changes in the gene expression of C. neoformans H99 cells exposed to FLC (Continued)

\begin{tabular}{|c|c|c|c|c|}
\hline 06574 & APP1 & & Antiphagocytic protein 1 & +2.21 \\
\hline 06482 & & AMD2 & Amidase & +2.20 \\
\hline 01252 & & TUM1 & Thiosulfate sulfurtransferase & -2.05 \\
\hline 03452 & & $A F G 1$ & AFG1 family mitochondrial ATPase & -2.16 \\
\hline 05831 & & MMF1 & Brt1 & -2.19 \\
\hline 03991 & & YGR149W & Integral to membrane protein & -2.39 \\
\hline 02039 & & YPL264C & Integral membrane protein & -2.46 \\
\hline 02943 & & SLM1 & Cytoplasm protein & -2.49 \\
\hline 06668 & & AIM38 & Mitochondrion protein & -2.61 \\
\hline 00638 & & LSGI & GTPase & -2.89 \\
\hline 01653 & $C I G$ & & Cytokine inducing-glycoprotein & -3.26 \\
\hline 04314 & & YEF1 & NAD + kinase & -3.74 \\
\hline 04690 & & FMP41 & Mitochondrion protein & -5.52 \\
\hline
\end{tabular}

Genes that were found to be differentially expressed were ordered by expression level and categorized, if available, into functional groups as described in Materials and Methods. Results are presented as the mean fold-increase (symbol + ) or -decrease (symbol -) of biological triplicates. Abbreviations: $C$. $n$., $C$. neoformans; S. c., S. cerevisiae.

[32], whose overexpression has been associated with fungal resistance [33]. To further classify the genes regulated by FLC exposure, we performed GO term analysis. As expected, GO analysis of genes induced by FLC revealed a significant enrichment of genes involved in sterol metabolism, particularly ergosterol biosynthetic process (Table 2). Enrichment of genes repressed by FLC was observed in processes involving metabolism of amino acids and derivatives (Table 2).

Effect of FLC on genes involved in ergosterol biosynthesis and related pathways

Earlier efforts to profile the response of yeast cells (S. cerevisiae or C. albicans) to the short-term exposure

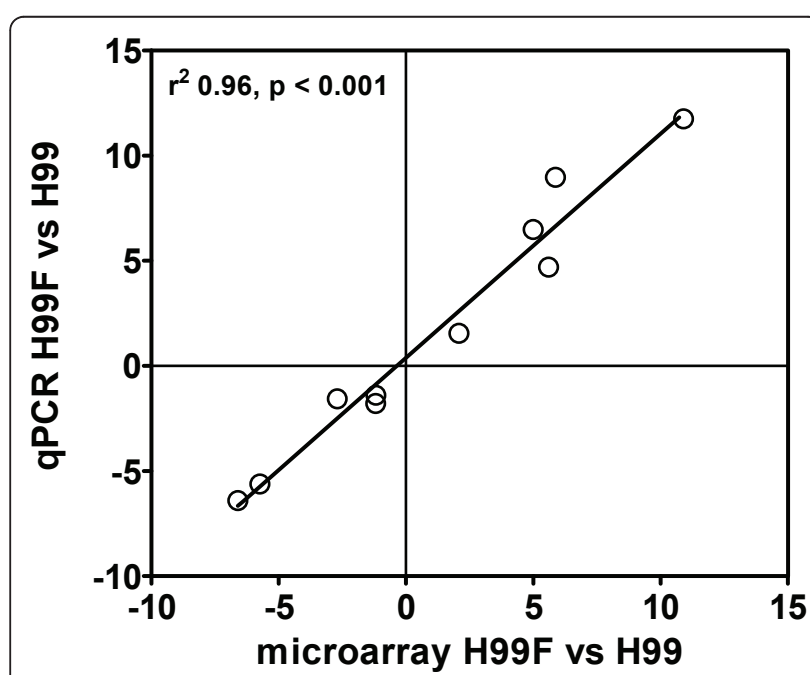

Figure 1 Scatter plot of the results by microarray and quantitative RT-PCR analyses for ten selected differentially regulated genes in $\mathrm{H} 99$ cells FLC-treated (H99F) compared to untreated control cells. to azole drugs implicated genes in the ergosterol biosynthetic pathway as major players [28,29], thus indicating that this pathway is the target of azoles and is responsive to modulations in ergosterol levels. As shown in Table 1, we found that eight ERG genes (ERG1, ERG2, ERG3, ERG5, ERG7, ERG11, ERG13 and ERG25) exhibited increases in expression (2.09- to 3.95-fold) upon FLC treatment. This was a predictable result from the inhibition of Erg11 function by FLC, which is the rate-limiting step of the ergosterol biosynthetic pathway. Indeed, the idea of a compensatory response to re-establish the plasma membrane ergosterol levels [30] may account for the observed upregulation of either early (ERG13, ERG7 and ERG1) or late (ERG25, ERG2, ERG3 and ERG5) genes

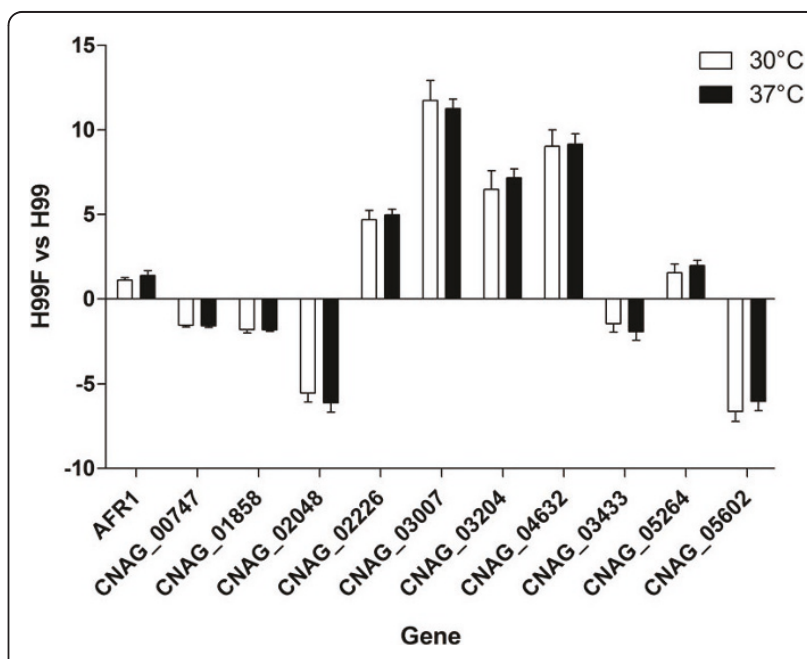

Figure 2 Results of qRT-PCR analysis performed with RNAs extracted from $\mathrm{H} 99$ cells FLC-treated (H99F) at $30^{\circ} \mathrm{C}$ and $37^{\circ} \mathrm{C}$.

The values, which are means of three separated experiments,

represent the increase in gene expression relative to untreated control cells (set at 1.00). Error bars show standard deviations 
Table 2 Gene Ontology (GO) term analysis for the C. neoformans FLC response

\begin{tabular}{|c|c|c|}
\hline GO group & GO subgroup & $P$-value \\
\hline \multicolumn{3}{|l|}{ Up-regulated genes } \\
\hline Oxidation reduction & & $5.26 \mathrm{e}^{-10}$ \\
\hline \multirow[t]{3}{*}{ Small molecule metabolic process } & & $1.34 e^{-06}$ \\
\hline & Alcohol metabolic process & $4.74 \mathrm{e}^{-07}$ \\
\hline & Sterol metabolic process & $4.41 e^{-07}$ \\
\hline \multirow[t]{4}{*}{ Steroid metabolic process } & & $7.81 e^{-07}$ \\
\hline & Phytosteroid metabolic process & $1.47 e^{-09}$ \\
\hline & Steroid biosynthetic process & $9.08 e^{-07}$ \\
\hline & Ergosterol biosynthetic process & $3.57 e^{-08}$ \\
\hline Transmembrane transport & & 0.00076 \\
\hline \multicolumn{3}{|l|}{ Down-regulated genes } \\
\hline Oxidation reduction & & $1.31 e^{-12}$ \\
\hline \multirow[t]{5}{*}{ Small molecule metabolic process } & & $2.50 e^{-11}$ \\
\hline & Alcohol metabolic process & 0.00037 \\
\hline & Cellular ketone metabolic process & $1.25 e^{-08}$ \\
\hline & Cellular amino acid and derivative metabolic process & $3.74 \mathrm{e}^{-12}$ \\
\hline & Organic acid metabolic process & $1.63 e^{-08}$ \\
\hline \multirow[t]{2}{*}{ Amine metabolic process } & & $1.47 e^{-13}$ \\
\hline & Gamma-aminobutyric acid metabolic process & 0.00078 \\
\hline
\end{tabular}

$\mathrm{GO}$ term assignment for $C$. neoformans $\mathrm{H} 99$ genes was based on homology to $S$. cerevisiae genes. $P$-value represents the probability that a particular GO term is enriched in the microarray gene list. The $P$-value cut-off was $<0.05$.

of the ergosterol pathway, in addition to upregulation of ERG11 itself (Table 1, ergosterol biosynthesis).

ERG13 encodes the enzyme hydroxymethylglutaryl-CoA synthase that catalyzes the production of hydroxymethylglutaryl-CoA from acetyl-CoA and acetoacetyl-CoA, and acts in the mevalonate biosynthesis, a precursor required for the biosynthesis of ergosterol. Acetyl-CoA is converted to carbon dioxide and water by enzymes (e.g. isocitrate dehydrogenase) that function in the TCA cycle, a central metabolic process in the mitochondria leading to produce, after oxidative phosphorylation, chemical energy in the form of ATP and NADH. Presumably, as a result of feedback control, we observed that several TCA cycle enzymes were downregulated in response to FLC (Table 1, TCA cycle), suggesting that $C$. neoformans may direct the cellular acetyl-CoA content to lipid (sterol) biosynthesis and metabolism to counterbalance ergosterol alteration.

Our particular interest was the up-regulation (4.04-fold) of SRE1, that belongs to a group of sterol regulatory element-binding proteins (SREBPs), first characterized in mammalian cells as regulator of lipid homeostasis [34]. While C. neoformans Sre1 regulates genes encoding ergosterol biosynthetic enzymes, SRE1 was shown to be required for growth and survival in the presence of azoles and also for virulence in a mouse model of cryptococcosis $[18,20,35]$. In addition, C. neoformans Sre1 stimulates ergosterol production in response to sterol depletion when the oxygen-dependent ergosterol synthesis is limited by hypoxia [36]. Consistently, C. neoformans mutants in the
SREBP pathway showed reduction in ergosterol levels, increased sensitivity not only to low oxygen but also to several chemical agents, including azole antifungals, $\mathrm{CoCl}_{2}$ and reactive oxygen species (ROS)-generating compounds. Most importantly, these mutants showed reduced virulence in mice [37].

\section{Effect of FLC on genes involved in cell structure and maintenance}

Consequent to depletion of ergosterol and the concomitant accumulation of 14-methylated sterols, several plausible hypotheses on the mode of action of azoles were suggested by Vanden Bossche [32] two decades ago including alterations in membrane functions, synthesis and activity of membrane-bound enzymes, mitochondrial activities and uncoordinated activation of chitin synthesis. Transcript levels of several genes involving lipid and fatty acid metabolism decreased in the current study (Table 1), possibly in agreement with a remodelling of the cell membrane in response to reduced ergosterol levels. Conversely, expression of PLB1, that encodes Plb1, a known virulence factor in $C$. neoformans, was increased 2.18-fold. Phospholipases cleave fatty acid moieties from larger lipid molecules, releasing arachidonic acid for the production of eicosanoids that are utilized by the pathogenic yeasts $C$. neoformans and $C$. albicans to produce immunomodulatory prostaglandins [38]. In addition, cell wall-linked cryptococcal Plb1 contributes to cell wall integrity and is a source of secreted enzyme [39]. 
It was also expected that exposure to FLC would affect genes responsible for cell wall integrity. Two chitin synthase genes were found to be significantly up-regulated (2.20-fold for CHS2 and 3.62-fold for CHS7), concomitantly with down-regulated expression (4.35-fold) of the chitin deacetylase $C D A 3$ (homolog to $S$. cerevisiae CDA2) (Table 1, cell wall maintenance). In C. albicans, activation of chitin synthesis, which is mediated by the PKC-, $\mathrm{Ca}^{2}$ +/calcineurin-, and HOG- cell wall signalling pathways, appears to be an adaptive response to caspofungin treatment. Hence, subculturing caspofungin-resistant cells in the absence of caspofungin resulted in wild-type levels of chitin content [40]. While this form of drug tolerance is rationally accepted for a drug damaging the cell wall integrity (caspofungin is known to reduce $\beta$-glucan synthesis), it is also possible that exposure to azoles induces a salvage mechanism involving the up-regulation of chitin synthesis. Although known as a relatively minor cell wall component, chitin is thought to contribute significantly to cryptococcal wall strength and integrity [3]. Chitosan, the enzymatically deacetytaled form of chitin, helps to maintain cell integrity and is necessary for maintaining normal capsule width and retention of cell wall melanin [41]. Consistently, up-regulation was observed for BGL2 (2.61-fold) that encodes the glucantransferase (also termed glucosyltransferase) Bgl2, a major cell wall constituent described in a wide range of yeast species.

\section{Effect of FLC on genes involved in cell stress and virulence}

We found that FLC induced the expression of several genes involved in oxidative-stress response (Table 1, cell stress). One of these genes, GRE2, was induced 3.54-fold, consistent with the previous observation that transcripts from GRE2 and other stress-induced genes (YDR453C and $S O D 2)$ were increased in $S$. cerevisiae exposed to azoles [28]. Interestingly, loss of Gre2 is impairing tolerance to ergosterol biosynthesis disrupting agents (i.e. clotrimazole and ketoconazole), further supporting an association between GRE2 and ergosterol metabolism [42]. YHB1 that encodes a flavo-haemoglobin able to detoxify nitric oxide in C. albicans and C. neoformans was down-regulated 2.32-fold in our study, which is opposed to its established relevance in vivo [43]. A strong reduction in the expression of FHB1 (the C. neoformans ortholog of YHB1) was also observed during growth of $C$. neoformans at $37^{\circ} \mathrm{C}$ compared to $25^{\circ} \mathrm{C}$, indicating that regulation of this gene or its product at the posttranslational level may occur in response to environmental changes [44]. In contrast, CTA1 encoding catalase in S. cerevisiae was induced (2.81fold) by FLC exposure. Together with TSA3 (2.09-fold) encoding thiol-specific antioxidant protein 3 (Table 1, cell stress) and other responsive genes with oxidoreductase activity (Table 1, oxidoreduction), these genes may function in response to oxidative stress. Accordingly, the stress-related gene encoding Ssa1 was also up-regulated (2.48-fold). This C. neoformans protein (Hsp70 family member) acts in vivo as transcriptional co-activator of laccase [45] and is important for the production of melanin, which is a free-radical scavenger playing a protective role in stress resistance [17].

The C. neoformans polysaccharide capsule is a complex structure that is required for virulence $[46,47]$. Interestingly, the capsule-associated gene CAS3 [48] was found to be up-regulated (12.16-fold) upon exposure to the drug (Table 1 , capsule synthesis). This gene encodes a protein belonging to a seven-member protein family that includes Cap64. Treatment with FLC did not significantly change expression of the essential capsule-producing genes, CAP10, CAP59, CAP60 and CAP64. Since the cryptococcal cell wall is needed for the localization or attachment of known or putative virulence factors other than capsule (i.e. melanin, Plb1 and Bgl2), it could be hypothesized that FLC induces alterations in the cell wall which in turns affects the expression of these factors. An alternative hypothesis would be that FLC acts as a stressgenerating molecule and triggers enhanced expression of virulence determinant(s) that enable to survive in hostile environments.

\section{Effect of FLC on genes involved in cellular transport}

Several genes involved in small molecule transport and vesicular transport were either up- or down-regulated in response to FLC (Table 1, transport). These include DUR3 (plasma membrane transporter for urea, up-regulated by 4.78-fold), MEP2/AMT2 (ammonium permease, upregulated by 3.78 -fold) and $A Q Y 1$ (aquaporin water channel, up-regulated by 2.73-fold), which all belong to the group of $C$. neoformans genes regulated by osmotic stress [49]. It is possible that defects in the plasma membrane resulting from inhibition of ergosterol biosynthesis by FLC affects transport of small molecules through the membrane. Analysis of the $\mathrm{H} 99$ genome sequence [16] predicted 54 ATP-Binding Cassette (ABC) transporters and 159 major facilitator superfamily (MFS) transporters, suggesting wide transport capabilities of this environmental yeast [50]. However, we found only two S. cerevisiae transporter homologues with significant increased expression. One is $P D R 15$ that is a member of the ABC transporter subfamily exporting antifungals and other xenobiotics in fungi [51]. The other gene is ATR1 that encodes a multidrug resistance transport protein belonging to the MFS class of transporters. ATR1 expression was recently shown to be upregulated by boron and several stress conditions [52]. To date, Afr1 (encoded by AFR1; also termed CneAfr1) and CneMdr1 are the only two efflux pumps associated with antifungal drug resistance in $C$. neoformans [50]. Since Afr1 is the major efflux pump mediating 
azole resistance in $C$. neoformans $[11,15]$, the absence of altered AFR 1 expression could be expected. Not surprisingly, we noticed downregulated expression (2.35-fold) of FLR1 (for fluconazole resistance) encoding a known MFS multidrug transporter in yeast, that is able to confer resistance to a wide range of dissimilar drugs and other chemicals [53]. This may suggest that both AFR1 and FLR1 do not participate to the short-term stress induced by FLC in C. neoformans.

\section{Effect of FLC on the susceptibility to cell wall inhibitors} It was demonstrated that compounds interfering with normal cell wall formation (Congo red, calcofluor white, SDS and caffeine) affect growth of C. neoformans strains with altered cell wall integrity [27]. For instance, several deletion strains for genes involved in the PKC1 signal transduction pathway were found to be sensitive to SDS and Congo red and to a lesser extent caffeine. To test the hypothesis that FLC treatment might induce cell wall stress, we analyzed $\mathrm{H} 99$ cells for susceptibility to the cell wall perturbing agents, before and after the cells were exposed for 90 min to FLC at sub-MIC concentration $(10 \mathrm{mg} / \mathrm{l})$ at $30^{\circ} \mathrm{C}$. Phenotypes of $\mathrm{H} 99$ cells on cell wall inhibitor plates are shown in Figure 3. The FLC pre-treated H99 cells were slightly more resistant to all four cell wall inhibitors as compared to untreated cells. These findings are consistent with expression changes of cell wall associated genes identified in our microarray analysis. Particularly, since calcofluor white (which binds to chitin) disrupts the cell wall and Congo red (which binds to $\beta$-glucans) interferes with the cell wall biogenesis [27], the altered regulation of genes involved in the chitin (CHS2 and CHS7) and glucan (BGL2) synthesis may explain the phenotype of decreased susceptibility to cell wall stress exhibited by FLC-exposed cells. Similar results were obtained when $\mathrm{H} 99$ cells were pre-treated with $\mathrm{FLC}$ at $37^{\circ} \mathrm{C}$ (see Additional file 2).

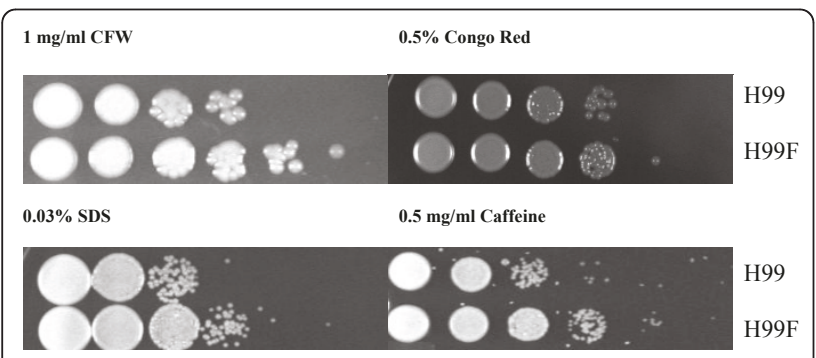

Figure 3 Cell wall integrity assays with H99 C. neoformans cells left untreated (H99) or exposed to FLC (H99F) at a sub-MIC concentration of $10 \mathrm{mg} / \mathrm{l}$ for $90 \mathrm{~min}$ at $30^{\circ} \mathrm{C}$. Cells were grown at the same temperature for $48 \mathrm{~h}$ on YEPD supplemented with calcofluor white (CFW), Congo red, sodium dodecyl sulphate (SDS) and caffeine. Aliquots of cells were applied onto the agar surface with 10 -fold serial dilutions.
Effect of FLC on the susceptibility to $\mathrm{H}_{2} \mathrm{O}_{2}$

Because a number of FLC-responsive transcriptional changes was found to affect genes involved in the oxidative stress response (i.e. CTA1, GRE2), it seemed reasonable to examine whether FLC at sub-inhibitory concentrations could induce oxidative stress resistance in vitro. For this purpose, exponentially growing $\mathrm{H} 99$ cells that were treated with $10 \mathrm{mg} / \mathrm{l} \mathrm{FLC} \mathrm{for} 90 \mathrm{~min}$ were subjected to an additional challenge with $20 \mathrm{mM} \mathrm{H}_{2} \mathrm{O}_{2}$. The viable cells were next quantified on YEPD plates after $0.5,1,1.5$ and $2 \mathrm{~h}$ of additional growth. As shown in Figure 4, while untreated cells showed a high degree of cell death, cells treated with FLC exhibited gained more viability upon oxidative exposure at the endpoints of 1 , 1.5 and $2 \mathrm{~h}$. Similar results were obtained when H99 cells were pre-treated with $\mathrm{FLC}$ at $37^{\circ} \mathrm{C}$ (see Additional file 3). These findings indicate that FLC exposure is able to generate protection against oxidative stress in vitro, possibly as a result of a transcriptional adaptive response.

\section{Conclusions}

Although exposure to azoles has been already investigated in several other fungal species and the transcriptional profile of differentially expressed genes was obtained using a single FLC concentration and time point, our study reveals several interesting findings. First, we demonstrated that short-term exposure of $C$. neoformans to FLC resulted in a complex altered gene expression profile. These genes included not only genes commonly responding to diverse environmental stresses, such as oxidative and drug stresses, but also genes encoding virulence factors (i.e. Plb1, Sre1 and capsule). Second, we corroborated the potential of genome-wide transcriptional analyses to envisage alternative therapeutic strategies for cryptococcosis. Apart from ergosterol and its biosynthesis, there are yet few other targets to be

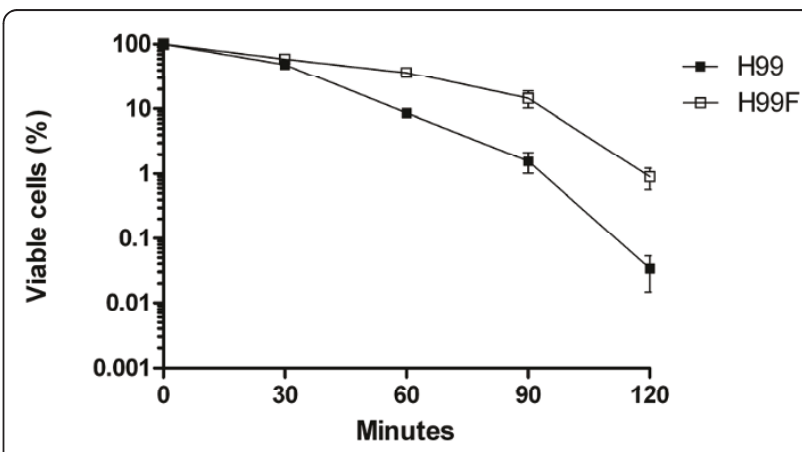

Figure 4 Survival of $C$. neoformans after oxidative treatment. Exponentially growing cells were left untreated $(\mathrm{H} 99)$ or exposed to $10 \mathrm{mg} / \mathrm{l} \mathrm{FLC} \mathrm{(H99F)} \mathrm{for} 90 \mathrm{~min}$ at $30^{\circ} \mathrm{C}$ and then challenged with 20 $\mathrm{mM} \mathrm{H}_{2} \mathrm{O}_{2}$ for $2 \mathrm{~h}$. Aliquots were harvested at given time points and cell viability performed as described in Methods. Plotted values are means of three experiments 
exploited in anticryptococcal therapy. Therefore, elucidation of molecular processes underlying the physiological responses of cryptococcal cells to FLC could serve not only to identify novel treatment approaches but also to potentiate the inhibitory effects of existing azole drugs. Our findings show that the phenomena described can apply to the in vivo situation, i.e. during azole maintenance therapy in the host, but transcriptional analyses using different growth conditions of H99 cells, mimicking stress conditions encountered during a human meningeal infection, may reveal new fields to pursue for anticryptococcal therapy.

\section{Additional material}

Additional file 1: Table A1 Primers and fluorescent probes used in qRT-PCR. Contains Table A1 showing the qRT-PCR primers and probes. Additional file 2: Figure A1 Cell wall integrity assays with $\mathrm{H} 99 \mathrm{C}$. neoformans cells left untreated (H99) or exposed to FLC (H99F) at a sub-MIC concentration of $10 \mathrm{mg} / \mathrm{l}$ for $90 \mathrm{~min}$ at $37^{\circ} \mathrm{C}$. Cells were grown at the same temperature for $48 \mathrm{~h}$ on YEPD supplemented with calcofluor white (CFW), Congo red, sodium dodecyl sulphate (SDS) and caffeine. Aliquots of cells were applied onto the agar surface with 10-fold serial dilutions. Contains Figure A1 showing the results of cell wall inhibitors susceptibility assays for $\mathrm{H} 99$ cells pre-treated with $\mathrm{FLC}$ at $37^{\circ} \mathrm{C}$.

Additional file 3: Figure A2 Survival of $C$. neoformans after oxidative treatment. Exponentially growing cells were left untreated (H99) or exposed to $10 \mathrm{mg} / \mathrm{FLC}$ (H99F) for $90 \mathrm{~min}$ at $37^{\circ} \mathrm{C}$ and then challenged with $20 \mathrm{mM} \mathrm{H}_{2} \mathrm{O}_{2}$ for $2 \mathrm{~h}$. Aliquots were harvested at given time points and cell viability performed as described in Methods. Plotted values are means of three experiments. Contains Figure A2 showing the results of $\mathrm{H}_{2} \mathrm{O}_{2}$ susceptibility assays for $\mathrm{H} 99$ cells pre-treated with $\mathrm{FLC}$ at $37^{\circ} \mathrm{C}$.

\begin{abstract}
Acknowledgements
This work was supported by grants from the Istituto di Ricovero e Cura a Carattere Scientifico (IRCCS) Lazzaro Spallanzani (Strategic Research Program 2006) to GF, from the Università Cattolica del S. Cuore (Fondi Ateneo Linea D1-2009) to MS, and from the Swiss Research National Foundation 31003A_127378 to DS

\section{Author details}

'Istituto di Microbiologia, Università Cattolica del Sacro Cuore, Largo F. Vito 1, 00168 Rome, Italy. ${ }^{2}$ Institute of Microbiology, University of Lausanne and University Hospital Center, Lausanne, Rue du Bugnon 48, CH-1011 Lausanne, Switzerland.
\end{abstract}

\section{Authors' contributions}

MS, DS and BP designed the study; ARF and SF carried out the experimental work; ARF, EDC and RT analysed the data; ARF and BP wrote the manuscript. GF and DS corrected the manuscript. All the authors read and approved the final manuscript.

Received: 22 December 2010 Accepted: 11 May 2011

Published: 11 May 2011

\section{References}

1. Perfect JR, Casadevall A: Cryptococcosis. Infect Dis Clin North Am 2002, 16:837-874.

2. Bicanic T, Harrison TS: Cryptococcal meningitis. Br Med Bull 2005, 72:99-118.

3. Doering TL: How sweet it is! Cell wall biogenesis and polysaccharide capsule formation in Cryptococcus neoformans. Annu Rev Microbiol 2009, 63:223-247.
4. Silveira FP, Husain S: Fungal infections in solid organ transplantation. Med Mycol 2007, 45:305-320.

5. Thakur R, Sarma S, Kushwaha S: Prevalence of HIV-associated cryptococcal meningitis and utility of microbiological determinants for its diagnosis in a tertiary care center. Indian J Pathol Microbiol 2008, 51:212-214.

6. Park BJ, Wannemuehler KA, Marston BJ, Govender N, Pappas PG, Chiller TM: Estimation of the current global burden of cryptococcal meningitis among persons living with HIV/AIDS. AIDS 2009, 23:525-530.

7. Datta K, Jain N, Sethi S, Rattan A, Casadevall A, Banerjee U: Fluconazole and itraconazole susceptibilities of clinical isolates of Cryptococcus neoformans at a tertiary care centre in India; a need for care. $J$ Antimicrob Chemother 2003, 52:683-686.

8. Sar B, Monchy D, Vann M, Keo C, Sarthou JL, Buisson Y: Increasing in vitro resistance to fluconazole in Cryptococcus neoformans Cambodian isolates: April 2000 to March 2002. J Antimicrob Chemother 2004, 54:563-565.

9. Bii CC, Makimura K, Abe S, Taguchi H, Mugasia OM, Revathi G, Wamae NC, Kamiya S: Antifungal drug susceptibility of Cryptococcus neoformans from clinical sources in Nairobi, Kenya. Mycoses 2007, 50:25-30.

10. Perfect JR, Cox GM: Drug resistance in Cryptococcus neoformans. Drug Resist Updat 1999, 2:259-269.

11. Sionov E, Chang YC, Garraffo HM, Kwon-Chung KJ: Heteroresistance to fluconazole in Cryptococcus neoformans is intrinsic and associated with virulence. Antimicrob Agents Chemother 2009, 53:2804-2815.

12. Mondon P, Petter R, Amalfitano G, Luzzati R, Concia E, Polacheck I, KwonChung KJ: Heteroresistance to fluconazole and voriconazole in Cryptococcus neoformans. Antimicrob Agents Chemother 1999, 43:1856-1861.

13. Sionov E, Lee H, Chang YC, Kwon-Chung KJ: Cryptococcus neoformans overcomes stress of azole drugs by formation of disomy in specific multiple chromosomes. PLoS Pathog 2010, 6:e1000848.

14. Lupetti A, Danesi R, Campa M, Del Tacca M, Kelly S: Molecular basis of resistance to azole antifungals. Trends Mol Med 2002, 8:76-81.

15. Posteraro B, Sanquinetti M, Sanglard D, La Sorda M, Boccia S, Romano L, Morace G, Fadda G: Identification and characterization of a Cryptococcus neoformans ATP binding cassette (ABC) transporter-encoding gene, CnAFR1, involved in the resistance to fluconazole. Mol Microbiol 2003, 47:357-371.

16. Loftus BJ, Fung E, Roncaglia P, Rowley D, Amedeo P, Bruno D, Vamathevan J, Miranda M, Anderson IJ, Fraser JA, Allen JE, Bosdet IE, Brent MR, Chiu R, Doering TL, Donlin MJ, D'Souza CA, Fox DS, Grinberg V, Fu J, Fukushima M, Haas BJ, Huang JC, Janbon G, Jones SJ, Koo HL, Krzywinski MI, Kwon-Chung JK, Lengeler KB, Maiti R, Marra MA, Marra RE, Mathewson CA, Mitchell TG, Pertea M, Riggs FR, Salzberg SL, Schein JE, Shvartsbeyn A, Shin H, Shumway M, Specht CA, Suh BB, Tenney A, Utterback TR, Wickes BL, Wortman JR, Wye NH, Kronstad JW, Lodge JK, Heitman J, Davis RW, Fraser CM, Hyman RW: The genome of the basidiomycetous yeast and human pathogen Cryptococcus neoformans. Science 2005, 307:1321-1324.

17. Brown SM, Campbell LT, Lodge JK: Cryptococcus neoformans, a fungus under stress. Curr Opin Microbiol 2007, 10:320-325

18. Chun CD, Liu OW, Madhani HD: A link between virulence and homeostatic responses to hypoxia during infection by the human fungal pathogen Cryptococcus neoformans. PLoS Pathog 2007, 3:e22.

19. Coleman JJ, Mylonakis E: Efflux in fungi: la pièce de résistance. PLoS Pathog 2009, 5:e1000486

20. Chang YC, Bien CM, Lee H, Espenshade PJ, Kwon-Chung KJ: Sre1p, a regulator of oxygen sensing and sterol homeostasis, is required for virulence in Cryptococcus neoformans. Mol Microbiol 2007, 64:614-629.

21. Eisenman HC, Casadevall A, McClelland EE: New insights on the pathogenesis of invasive Cryptococcus neoformans infection. Curr Infect Dis Rep 2007, 9:457-464.

22. Soteropoulos P, Vaz T, Santangelo R, Paderu P, Huang DY, Tamás MJ, Perlin DS: Molecular characterization of the plasma membrane $\mathrm{H}$ ${ }^{+}$-ATPase, an antifungal target in Cryptococcus neoformans. Antimicrob Agents Chemother 2000, 44:2349-2355.

23. Sanguinetti M, Posteraro B, La Sorda M, Torelli R, Fiori B, Santangelo $R$, Delogu G, Fadda G: Role of $A F R 1$, an $A B C$ transporter-encoding gene, in the in vivo response to fluconazole and virulence of Cryptococcus neoformans. Infect Immun 2006, 74:1352-1359.

24. Kim MS, Ko YJ, Maeng S, Floyd A, Heitman J, Bahn YS: Comparative transcriptome analysis of the $\mathrm{CO}_{2}$ sensing pathway via differential 
expression of carbonic anhydrase in Cryptococcus neoformans. Genetics 2010, 185:1207-1219.

25. Barrett ER: Gene Expression Omnibus (GEO): Microarray data storage, submission, retrieval, and analysis. Methods Enzymol 2006, 411:352-369.

26. Arana DM, Nombela C, Pla J: Fluconazole at subinhibitory concentrations induces the oxidative- and nitrosative-responsive genes TRR1, GRE2 and YHB1, and enhances the resistance of Candida albicans to phagocytes. $J$ Antimicrob Chemother 2010, 65:54-62.

27. Gerik KJ, Donlin MJ, Soto CE, Banks AM, Banks IR, Maligie MA, Selitrennikoff CP, Lodge JK: Cell wall integrity is dependent on the PKC1 signal transduction pathway in Cryptococcus neoformans. Mol Microbiol 2005, 58:393-408.

28. Bammert GF, Fostel JM: Genome-wide expression patterns in Saccharomyces cerevisiae: comparison of drug treatments and genetic alterations affecting biosynthesis of ergosterol. Antimicrob Agents Chemother 2000, 44:1255-1265.

29. De Backer MD, llyina T, Ma XJ, Vandoninck S, Luyten WH, Vanden Bossche $\mathrm{H}$ : Genomic profiling of the response of Candida albicans to itraconazole treatment using a DNA microarray. Antimicrob Agents Chemother 2001, 45:1660-1670.

30. Gamarra S, Rocha EM, Zhang YQ, Park S, Rao R, Perlin DS: Mechanism of the synergistic effect of amiodarone and fluconazole in Candida albicans. Antimicrob Agents Chemother 2010, 54:1753-1761.

31. Karababa M, Coste AT, Rognon B, Bille J, Sanglard D: Comparison of gene expression profiles of Candida albicans azole-resistant clinical isolates and laboratory strains exposed to drugs inducing multidrug transporters. Antimicrob Agents Chemother 2004, 48:3064-3079.

32. Vanden Bossche $\mathrm{H}$ : Biochemical targets for antifungal azole derivatives: hypothesis on the mode of action. Curr Top Med Mycol 1985, 1:313-351.

33. Henry KW, Nickels JT, Edlind TD: Upregulation of ERG genes in Candida species by azoles and other sterol biosynthesis inhibitors. Antimicrob Agents Chemother 2000, 44:2693-2700.

34. Goldstein JL, Debose-Boyd RA, Brown MS: Protein sensors for membrane sterols. Cell 2006, 124:35-46.

35. Bien CM, Chang YC, Nes WD, Kwon-Chung KJ, Espenshade PJ: Cryptococcus neoformans Site-2 protease is required for virulence and survival in the presence of azole drugs. Mol Microbiol 2009, 74:672-690.

36. Bien $C M$, Espenshade PJ: Sterol regulatory element binding proteins in fungi: hypoxic transcription factors linked to pathogenesis. Eukaryot Cell 2010, 9:352-359

37. Chang YC, Ingavale SS, Bien C, Espenshade P, Kwon-Chung KJ: Conservation of the sterol regulatory element-binding protein pathway and its pathobiological importance in Cryptococcus neoformans. Eukaryot Cell 2009, 8:1770-1779.

38. Rhome R, Del Poeta M: Lipid signaling in pathogenic fungi. Annu Rev Microbiol 2009, 63:119-131.

39. Siafakas AR, Sorrell TC, Wright LC, Wilson C, Larsen M, Boadle R, Williamson PR, Djordjevic JT: Cell wall-linked cryptococcal phospholipase B1 is a source of secreted enzyme and a determinant of cell wall integrity. J Biol Chem 2007, 282:37508-37514.

40. Walker LA, Gow NA, Munro CA: Fungal echinocandin resistance. Fungal Genet Biol 2010, 47:117-126.

41. Baker LG, Specht CA, Donlin MJ, Lodge JK: Chitosan, the deacetylated form of chitin, is necessary for cell wall integrity in Cryptococcus neoformans. Eukaryot Cell 2007, 6:855-867.

42. Warringer J, Blomberg A: Involvement of yeast YOL151W/GRE2 in ergosterol metabolism. Yeast 2006, 23:389-398.

43. de Jesús-Berríos M, Liu L, Nussbaum JC, Cox GM, Stamler JS, Heitman J: Enzymes that counteract nitrosative stress promote fungal virulence. Curr Biol 2003, 13:1963-1968.

44. Kraus PR, Boily MJ, Giles SS, Stajich JE, Allen A, Cox GM, Dietrich FS, Perfect JR, Heitman J: Identification of Cryptococcus neoformans temperature-regulated genes with a genomic-DNA microarray. Eukaryot Cell 2004, 3:1249-1260.

45. Zhang S, Hacham M, Panepinto J, Hu G, Shin S, Zhu X, Williamson PR: The Hsp70 member, Ssa1, acts as a DNA-binding transcriptional co-activator of laccase in Cryptococcus neoformans. Mol Microbiol 2006, 62:1090-1101.

46. Fromtling RA, Shadomy HJ, Jacobson ES: Decreased virulence in stable, acapsular mutants of Cryptococcus neoformans. Mycopathologia 1982, 79:23-29.
47. Chang YC, Kwon-Chung KJ: Complementation of a capsule-deficient mutation of Cryptococcus neoformans restores its virulence. Mol Cell Biol 1994, 14:4912-4919.

48. Moyrand F, Chang YC, Himmelreich U, Kwon-Chung KJ, Janbon G: Cas3p belongs to a seven-member family of capsule structure designer proteins. Eukaryot Cell 2004, 3:1513-1524.

49. Ko YJ, Yu YM, Kim GB, Lee GW, Maeng PJ, Kim S, Floyd A, Heitman J, Bahn YS: Remodeling of global transcription patterns of Cryptococcus neoformans genes mediated by the stress-activated HOG signaling pathways. Eukaryot Cell 2009, 8:1197-1217.

50. Cannon RD, Lamping E, Holmes AR, Niimi K, Baret PV, Keniya MV, Tanabe K, Niimi M, Goffeau A, Monk BC: Efflux-mediated antifungal drug resistance. Clin Microbiol Rev 2009, 22:291-321.

51. Seret ML, Diffels JF, Goffeau A, Baret PV: Combined phylogeny and neighborhood analysis of the evolution of the $A B C$ transporters conferring multiple drug resistance in hemiascomycete yeasts. $B M C$ Genomics 2009, 10:459.

52. Kaya A, Karakaya HC, Fomenko DE, Gladyshev VN, Koc A: Identification of a novel system for boron transport: Atr1 is a main boron exporter in yeast. Mol Cell Biol 2009, 29:3665-3674.

53. Sá-Correia I, dos Santos SC, Teixeira MC, Cabrito TR, Mira NP: Drug:H+ antiporters in chemical stress response in yeast. Trends Microbiol 2009, 17:22-31.

doi:10.1186/1471-2180-11-97

Cite this article as: Florio et al: Genome-wide expression profiling of the response to short-term exposure to fluconazole in Cryptococcus neoformans serotype A. BMC Microbiology 2011 11:97.

\section{Submit your next manuscript to BioMed Central and take full advantage of:}

- Convenient online submission

- Thorough peer review

- No space constraints or color figure charges

- Immediate publication on acceptance

- Inclusion in PubMed, CAS, Scopus and Google Scholar

- Research which is freely available for redistribution 